gesehen haben, offenbar darin zu suchen, dass beide Erscheinungsgruppen sich ohne die unmittelbare Mitwirkung der dieselben ursprünglich hervorrufenden Ursachen geltend machen. Die Nachwirkungen sind unzweifelhaft Folge äusserer Einflüsse, denen der Organismus zu einer bestimmten Zeit ausgesetzt gewesen war; viele erblich gewordene Charaktere der Pflanzen sowie Thiere sind sicher ebenfalls durch äussere Einflüsse inducirt. We is mann's Anschauungen erweisen sich demnach auch unter Beriicksichtigung der zuletzt geltend gemachten Gesichtspunkte als nicht genuigend begriindet.

Jena, im Juni 1887.

(Aus dem physiologischen Institut der Universität Strassburg.)

\title{
Aendert sich das Volumen eines Muskels bei der Contraction?
}

Von

Prof. J. Rich. Ewald.

Hierzu 2 Holzschnitte.

Diese Frage ist in älterer und neuerer Zeit ${ }^{1}$ ) vielfach untersucht worden, ohne zu einem befriedigenden Abschluss gekommen zu sein. Im Allgemeinen hat jeder spätere Beobachter das entgegengesetzte Resultat von dem seines Vorgängers erhalten. Da aber immer neue Methoden zur Untersuchung verwendet worden sind, so wäre es ja möglich, dass die Verschiedenheit der Resultate auf die verschiedene Feinheit der Untersuchungsmethoden

1) Die einschlägige Literatur findet sich zusammengestellt in Hermann's Handbuch der Physiologie, Bd. I, Abthl. 1, p. 14 u. 15. 
zurückzufuihren wäre. Nachfolgende Zeilen werden indess diese Erklärung widerlegen, denn es findet auch bei Anwendung von bedeutend empfindlicheren Methoden als den bisher benutzten keine nachweisbare Volumenverminderung statt.

Wie gross die Empfindlichkeit war, die die früleren Autoren mit ihren Methoden erreicht haben, lässt sich freilich nicht genau feststellen - es fehlten die nöthigen Angaben über die Dimensionen der Apparate - aber sie war keinenfalls auch nur annähernd so gross wie bei den entsprechenden von mir angestellten Versuchen. Da ich nun auch nicht die geringste Volumenverminderung wahrnehmen konnte, so blieb schliesslich nur noch der Einwand übrig, dass vielleicht meine Methode -- es war im Wesentlichen die Erman'sche - nicht ganz zuverlässig sei. In der That spielt bei allen bisher angewandten Methoden der Capillardruck eine gewisse und unter Umständen sicherlich eine störende Rolle; bei der Erman'schen Steigröhre in dieser selbst, bei Kühne's Aräometer und bei Valentin's Wage an den Stellen, wo der Aräometerbals oder der Coconfaden durch das Niveau der Flüssigkeit treten.

Nun bin ich zwar davon uberzeugt, dass diese Fehlerquelle in meinen eigenen Versuchen - erste Versuchsreihe - viel zu gering war, um auf das Resultat irgend welchen Einfluss zu haben, da aber der Beweis hierfür nur schwierig zu führen gewesen wäre, so stellte ich noch eine zweite Versuchsreihe mit einer neuen Methode an, die jedenfalls von genannter Fehlerquelle ganz frei ist. Diese Methode besteht darin, den völlig frei schwebenden Muskel $z \mathfrak{u}$ beobachten. Ihre Empfindlichkeit steht freilich hinter derjenigen meiner andern Versuche weit zuriick, immerhin ist sie wohl noch grösser als die von meinen Vorgängern erreichte und jedenfalls gross genug um für unser Bedürfniss an Genauigkeit die Frage zu entscheiden.

Der Muskel nimmt also bei seiner Contraction nicht an Volumen ab. Wenn aber dem so ist und daher die Empfindlichkeit der Untersuchungsmethode nieht mehr zur Erklärung herangezogen werden kann, wie sind dann die abweichenden Resultate der frïheren Autoren möglich gewesen? Es erscheint vollständig räthselhaft, dass Erman, Marchand, Ed. Weber und Valentin eine Volumenabnahme - und noch dazu eine recht grosse - beobachten konnten. Joh. Müller hat die Vermuthung ausgesprochen, es 
könnte sich vielleicht um Compression von Luft im Innern des Muskels handeln. Aber abgesehen davon, dass ich diesen Einwand wie unten ausführlich gezeigt werden soll, nicht gelten lassen möchte, ist diese Fehlerquelle durch das Schlachten der Thiere unter ausgekochtem Wasser von Marchand und Ed. Weber ganz ausgeschlossen worden.

Eine andere Erklärung ist nicht zu finden. Denn bei der grossen Einfachheit und Uebersichtlichkeit der Versuche dürfen wir weder an Beobachtungsfebler noch an eine mangelhafte Versuchsanordnung denken und wenn wir schliesslich die Fehlerquellen, auf die man allenfalls verfallen könnte, durchgehen, so finden wir, dass sie immer nur eine Volumenzunahme aber keine Abnahme vortäuschen würden.

Da es mir nun wichtig schien die Ursache dieser merkwilrdigen Widersprüche aufzudecken, so babe ich auch die Versuche von Erman, Ed. Weber und Valentin in der Weise wiederholt, dass ich mich möglichst genau an ihre Angaben hielt. Es glückte mir auch wirklich Feblerquellen zu finden, die die abweichenden Resultate von Erman, Marchand und Weber erklären können, und damit ist, glaube ich, in diesem Falle schon etwas gewonnen.

Beobachtung des Niveaus in einer Steigröhre.

Diese Methode wandten eigentlich schon die ältesten Forscher, wie Swammerdam, Glisson u. s. w. an, nur dass sie noch keine Steigröbre benutzten, sondern das Niveau der Flüssigkeit in dem Gefäss selbst beobachteten. Natürlich wird aber die Methode erst fitr genauere Bestimmungen brauchbar, wenn man sich wie Erman des grossen Vortheils einer Steigröhre bedient.

Ich halte diese Methode für die bei Weitem empfindlichste, wenn man sie in richtiger Weise und unter günstigen Bedingungen (was freilich Erman und seine Nachfolger nicht gethan haben) zur Ausfuibrung bringt. Es ist klar, dass die Beobachtung der Niveauveränderungen desto genauer wird, je enger die Steigröhre ist und man muss sie daher zu einer Capillare ausziehen. Diesen Vortheil hat sich Ermann entgehen lassen indem er den einen Zuleitungsdraht durch die Steigröhre selbst zog. Denn wenn man auch auf diese Weise dem Steigraum einen sehr kleinen Querschnitt geben könnte indem man die Dicke des Drahtes so wählt, 
dass er beinahe das ganze Lumen der Röhre ausfüllt, so würde man dadurch doch keinen Vortheil haben, denn es würde nun der Steigraum von der regelmässig cylindrischen Gestalt des Drahtes abhängig und miisste entsprechend jeder Biegung dieses letzteren verschiedene Formen annehmen. Mit einer Capillare erreicht man überdies sehr leicht einen so kleinen Querschnitt, dass, wenn man nach Erman's Vorgang verfahren wollte, der durch die unvermeidlichen Unregelmässigkeiten in der Gestalt des Steigraums wechselnde Capillardruck zu einer jede Beobachtung störenden Fehlerquelle werden würde. Ein weiterer Fehler der Erman'schen Methode beruht in dem Verschluss des Gefässes durch einen einfachen Korkpfropfen, dessen Unbrauchbarkeit wir später noch ausführlich zu besprechen haben.

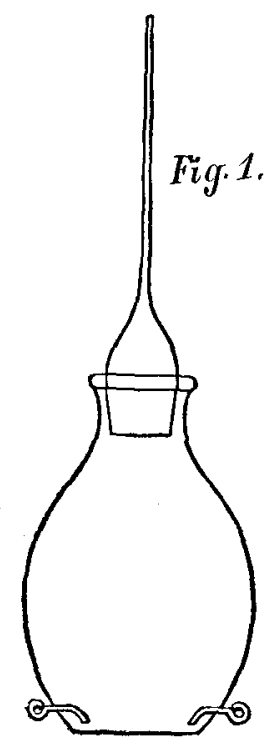

Figur 1.

Ich selbst habe diese Methode in folgender Weise angewandt. In ein kleines Glasfläschchen (Pyknometer) Figur I werden dicht über dem Boden zwei Platindrähte eingeschmolzen, so dass sie sich diametral gegenïberstehen. Sie reichen einige Millimeter weit in das Fläschchen hinein und sind darin nach abwärts gebogen. Aussen bilden sie kleine Oesen, in die man leicht die Leitungsdrähte einhängen kann, die zu einem Reizinductorium führen. Der eingeriebene Glasstopfen des Fläschehens ist hohl und endigt in einer kurzen dickwandigen Steigröbre. Sie wird zu einer feinen Capillare ausgezogen ${ }^{1}$ ).

Ich verwandte zu diesen Untersuchungen immer den Gastrocnemius eines Frosches. Das Thier wurde unter ausgekochtem Wasser getödtet und enthäutet. Das Wasser enthielt, wie in

1) Da ich gesehen habe, dass auch geübte Glasbläser in Verlegenheit gerathen können, wenn sie eine dickwandige Röhre mit einem engen Lumen zu einer Capillare ausziehen sollen, weil die Röhre immer zuschmilzt, so sei hier der eigentlich selbstverständliche Kunstgriff erwähnt, dass man zuerst die Röhre zu einer kleinen Kugel aufzublasen hat und dass man diese dann auszieht. 
allen Fällen, wenn in dieser Arbeit von ausgekochtem Wasser die Rede ist, 0,6 Proc. Kochsalz. Während der Frosch nun immer unter Wasser blieb, präparirte ich den Gastrocnemius frei und löste ihn schliesslich ganz vom Körper ohne den Nerv. ischiadicus mit zu nehmen. In das ebenfalls mit ausgekochtem Wasser gefullte Fläschchen gebracht, fällt der Muskel vermöge seines grösseren specifischen Gewichts zu Boden und kommt zwischen den Electroden zu liegèn. Das Fläschchen wird nun soweit aufgefüllt, dass sich das Wasser in einem Meniscus über den Rand wölbt. Dann ist noch der Glasstopfen zu füllen: Man zieht über die weite Oeffnung desselben ein Stïckchen Gummischlauch und saugt, während man die Oeffnung der Capillare unter Wasser hält, letztere und die Höhlung des Stopfens so weit voll, bis man mit einer Pipette den Rest bequem nachfüllen kann. Das Ueberziehen des Gummischlauchs hat dabei nur den Zweck, ein etwaiges Herabfliessen von Speichel in den Stopten zu verhindern. Der auf diese Weise ganz gefüllte Stopfen wird in das Fläschchen eingesetzt. Dies vollzog sich bei meinem Fläschchen ohne weitere Schwierigkeit, da beim langsamen Umdrehen des Stopfens keine Luftblase in denselben eindrang. Hat die Flasche aber einen etwas weiteren Hals, so muss man den gefüllten Stopfen zuerst mit einem Scheibchen Papier bedecken, das man dann fortzieht, wenn er sich ïber der Flaschenöffnung befindet. Es ist aber unter allen Umständen leicht das Eindringen auch des kleinsten Luftbläschens zu vermeiden.

War der Stopfen dann fest eingedrückt und waren die Leitungsschnüre in die Platinösen gehängt, so stellte ich das Fläschchen auf ein Stativ vor ein in horizontaler Lage befindliches Mikroskop (Schieck, Object. Nr. IV) mit einem Mikrometer-Ocular. Bevor der Versuch beginnen konnte, musste noch das Niveau der Fluissigkeit, welches sich am äussersten Ende der Capillare befand, in einen mittleren Theil derselben gebracht werden. Es geschah dies durch Aufträufeln einiger Tropfen Aether auf die Wandungen des Fläschchens, wodurch das Niveau so stark zum Steigen gebracht wird, dass ein kleines Flüssigkeitsquantum aus der Capillare herausläuft resp. mit Fliesspapier aufgesogen werden kann und wenn sich unmittelbar darauf das Glas des Fläschehens wieder ausdehnt, so steht nun das Niveau in einem für die Beobachtung günstigen Abschnitt der Capillare. Nach kurzer Zeit bewegt sich 
die Flïssigkeit nicht mehr oder wenigstens so langsam, dass die Untersuchung dadurch nicht gestört wird. Ist dann auch das Mikroskop genau eingestellt, so kann man nun den Muskel zur Contraction bringen, während man gleichzeitig das Niveau durch das Mikroskop beobachtet. Die für diese Vorbereitungen nöthige Zeit, von dem Moment der Köpfung des Frosches bis zur Bewegung des den Reiz vermittelnden electrischen Schlüssels, beträgt etwa 3 Minuten.

Bei keinem der zahlreich angestellten Versuche konnte ich das geringste Schwanken des Niveaus in der Capillare wahrnehmen.

\section{Empfindlichkeit der Methode.}

Nähert man dem Fläschchen dic flache Hand auf einige Centimeter, während man das Niveau durch das Mikroskop beobachtet, so sieht man die Flüssigkeit mit grosser Geschwindigkeit sinken. Es rührt dies daher, dass durch die strahlende Würme zuerst das Glas ausgedehnt wird. Erst nach einiger Zeit macht sich die Wirkung der Wärme auf die Flïssigkeit durch ein nunmehriges Steigen derselben bemerkbar. Die umgekehrte Wirkung bei der Abkïhlung des Glases dureh einige Tropten Aether haben wir bereits besprochen.

Wenn das Nivean in Folge einer allmählichen Erwärmung der Flüssigkeit langsam steigt, so kann man sich davon überzeugen, dass nirgends ein Stocken, nirgends eine plötzliche Steigerung der Geschwindigkeit eintritt. Wenn man aber das Mikroskop auf das Ende der Capillare gerichtet hat und durch dasselbe das allmähliche Steigen des Niveaus beobachtet, so sieht man in dem Moment, wenn die Fliissigkeit das Ende der Capillare erreicht, eine merkwiurdige Aenderung in ibrem Verhalten eintreten. Ganz gleichmässig fortschreitend ist sie bis zum Ende der Capillare gestiegen, nun aber tritt plötzlich völliger Stillstand ein und kein kleines Tröpfehen tritt, wie man erwarten sollte, aus der Capillare hervor. Wie geht das zu? Hat so plötzlich die Erwärmung der Fliussigkeit aufgehört? Natürlich nicht, sondern der Capillardruek wirkt nun dem Austreten der Flüssigkeit entgegen, und da in Folge davon der Druck im Innern des Fläschchens steigt, dehnen sich seine Wände ans, bis endlich der Druck genïgend gross geworden ist um den Capillardruck zu iuberwinden. Jetzt erst wölbt sich das 
erste Tröpfchen über die Oeffnung der Capillare. Man sieht, dass dieses Experiment, welches man nach dem negativen Ausfall der Beobachtung des Muskelvolums anstellen mag ohne das Fläschchen inzwischen berïhrt zu haben, die Garantie dafür giebt, dass der Stopfen dicht und fest im Fläschchen bält.

Will man sich jetzt andrerseits noch besonders davon überzeugen, dass sich auch eine kleine und plötzliche Volumzunahme im Innern des Fläschchens durch die Beobachtung des Niveaus erkennen lässt, so schiebt man die secundäre Spirale des Inductoriums so weit über die primäre, bis man eine vorher genau bestimmte Stellung erreicht, bei der eine ganz schwache Gasentwickelung an den Electroden beginnt. Durch einen kurzdauernden Schluss des electrischen Stroms bekommt man auf diese Weise eine plötzliche ganz kleine Volumenzunahme, von deren Grösse man sich ungefähr eine Vorstellung machen kann, wenn man die auf den Electroden befindlichen eben nur sichtbaren Gasbläschen ibrer Grösse nach schätzt. Mit einem solchen kurzdauernden Kettenschluss ist ein Aufschiessen des Niveaus iiber das ganze Gesichtsfeld verbunden. Vor dem Versuch war natürlich das Niveau wieder in einen mittleren Abschnitt der Capillare gebracht worden.

Die Capillare war innerhalb der Strecke, die zu den maassgebenden Beobachtungen benutzt wurde, sehr gleichmässig weit und völlig rund auf dem Querschnitt. Am oberen Ende dieses etwa $2 \mathrm{~cm}$ langen Stückes hatte ihr Lumen einen Durchmesser von $0,1417 \mathrm{~mm}$, am unteren Ende betrug er 0,1474 mm. Der äussere Durchmesser war dabei etwa $0,32 \mathrm{~mm}$ gross. Es entsprach ferner ein Theilstrich des Mikrometeroculars $0,0075 \mathrm{~mm}$. Eine Bewegung des Niveaus um einen balben Theilstrich hätte mit grösster Leichtigkeit erkannt werden müssen, auch dann, wenn sich zu gleicher Zeit das Niveau nicht in völliger Ruhe, sondern in langsamem Steigen oder Sinken befand. So erhalten wir als grösstes Volumen, welches mit Sicherheit der Muskel bei der Contraction (Tetanus) nicht verloren hat, einen Kreiscylinder von $0,00375 \mathrm{~mm}$ Höhe und einem mittleren Durchmesser von $0,14455 \mathrm{~mm}$. Dieses Volumen beträgt 0,00006 Cubmm. Der Muskel hat also sicher kein Zehntausendstel eines Cubikmillimeters an Inhalt verloren ${ }^{1}$ ).

1) Bei einer so ausserordentlich grossen Empfindlichkeit der Methode muss man sich fragen, ob nicht bereits Fehlerquellen von sonst verschwindender 
Die Methode einen in Flüssigkeit schwebenden Muskel zu beobachten.

Das alte Experiment eine Oelkugel in Flüssigkeit schweben $\mathrm{za}$ lassen, wird bekanntlich so ausgeftihrt, dass man das Oel unter verdünnten Alkohol bringt. Durch Hinzufügen von Alkohol oder von Wasser kann man das specifische Gewicht der Mischung leicht demjenigen des Oels genau gleich machen und in Folge davon schwebt das Oel in der Mitte der Flüssigkeit. Offenbar kann man die schwebende Oelkugel wie ein Aräometer benutzen. Wir werden zunächst aus der Stellung, die sie in der Flüssigkeit einnimmt, auf das specifische Gewicht der letzteren schliessen können. Betrachten wir die Oelkugel durch ein Fernrohr, so sehen wir sie ganz langsam steigen, weil sich das specifische Gewicht der Flüssigkeit allmählich vergrössert. Denn indem der Alkohol schneller verdunstet als das Wasser wird die Mischung allmählich schwerer. Wir brauchen nur das Gefäss luftdicht zu verschliessen, dann bleibt auch die Kugel unverrickt an ibrem Platze.

Ferner können wir aber auch aus der Lage der Kugel auf das specifische Gewicht des Oels schliessen, wenn dasjenige der umgebenden Flüssigkeit constant bleibt. Hat man drei gleiche Portionen Oel, und hat man die erste Portion rein gelassen, während man zur zweiten wenige Tropfen Terpentinöl gemischt hat und zur dritten noch einige Tropfen mehr als zur zweiten und bringt man dann von jeder der drei Portionen Oel etwas in verdïnnten Alkohol, dessen specifisches Gewicht gleich dem der zweiten Oelportion ist, so sieht man das reine Oel dicht über dem Boden schweben, die dritte Portion dicht unter der Oberfläche und die zweite in der Mitte. In ähnlicher Weise muss sich also auch die

Grösse eine Rolle spielen. Es wäre zunächst an die Erwärmung durch die elektrischen Ströme zu denken. Das negative Resultat des Versuchs kann allein nicht dafür bürgen, dass keine in Betracht kommende Ausdehnung der Flüssigkeit oder der eingeschmolzenen Drähte statt fand, denn diese Volumenszunahme konnte ganz oder theilweise durch eine gleichzeitige Abnahme des Muskelvolumens compensirt worden sein. Die betreffenden Fehlerquellen werden aber für unsern Versuch ausgeschlossen, wenn man die electrischen Ströme durch die Flüssigkeit schickt, ohne dass sich ein Muskel in ihr befindet und dann ebenfalls keine Niveauveränderung beobachtet. Auch als ich die electrischen Ströme bedeutend stärker machte, als die später bei dem Versuch benutzten, sah ich noch keine direkte Wirkung derselben. 
Lage eines jeden schwebenden Körpers ändern, falls sein specifisches Gewicht durch irgendwelche Umstände zu- oder abnimmt.

Dies brachte mich auf den Gedanken einen Muskel in Flïssigkeit sehwebend zu erhalten und nachzusehen, ob er während der Dauer des Tetanus sinkt. Der Muskel ist dann selbst Aräometer, aber ein Aräometer, welches nicht durch das Niveau der Flässigkeit hindurch in die Luft ragt, so dass ein Einfluss des Capillardrucks ganz ausgeschlossen ist. Dies ist sein grosser Vorzug vor den von Kühne angewandten Aräometern. Gegenuiber der Valentin'schen Methode aber hat diese Anordnung ausser dem obengenannten noch den Vortheil, dass der Muskel bei seiner Bewegung nicht den Wagebalken mit sich zu ziehen braucht.

Die Versuche wurden folgendermaassen angestellt. In einer ersten Versuchsreihe kam der Gastrocnemius des Frosches zur Verwendung. Sein specifisches Gewicht betrug in einem Fall 1,0765 , in andern Fällen wich es nur wenig von dieser Zahl ab. Die Flüssigkeit, in der der Muskel schweben sollte, musste also ein gleiches specifisches Gewicht hahen. Es wird dies von einer Kochsalzlösung aber erst bei etwa 10,5\% erreicht, von einer ZuckerJösung erst bei etwa $18,5 \%$. Da diese Lösungen von so hoher Concentration die Erregbarkeit des Musekels schon stark beeinflussen, so musste von ihnen Abstand genommen werden. Uebrigens bestand ibr Hauptnachtheil darin, dass der Muskel in ihnen durch Diffusion schwerer wird, so dass er beständig sinkt und für die Dauer eines Versuchs nicht genïgend seine Lage in der Flüssigkeit beibehält. Ich wandte daher Gummilösungen an, indem ich die officinelle mucilago gummi mimosae, welche specifisch viel schwerer als der Muskel ist, mit destillirtem Wasser verdünnte. In einer solchen Lösung wird der Muskel ausgelaugt und daher allmählich leichter. Ein Zusatz von $0,6 \%$ Kochsalz beseitigt diesen Nachtheil.

Nachdem der Frosch unter ausgekochtem Wasser decapitirt $\left.{ }^{1}\right)$ war, wurde zunächst der eine Gastrociemius unter Wasser aus dem Körper entfernt und die Flüssigkeit auf sein specifisches Gewicht abgestimmt. Dieselbe befindet sich in einem graduirten

1) Von einer vollständigen Tödtung des Thiers wurde Abstand genommen, um die Erregbarkeit des zweiten Präparats, welches später dem Körper entnommen wurde, nicht zu.sehr herabzusetzen. 
Messcylinder, dessen Theilstriche den Vortheil gewähren, die Bewegung des Muskels besser wahrnehmen zu können, falls man sie direkt mit dem Auge und nicht durch ein Fernrohr beobachten will. Zur leichteren Abstimmung der Flüssigkeit hatte ich zwei Gummilösungen vorräthig, von denen die eine etwas zu schwer, die andere etwas zu leicht war. Es gewährt dies den Vortheil beim Abstimmen der Lösung nicht mit so kleinen Quanten operiren zu müssen, als wenn man reines Wasser oder andererseits die officinelle Gummilösung direct dazn verwenden würde. Dieser Vortheil ist wichtig, da es natïrlich auf ein schnelles Treffen des richtigen speeifischen Gewichtes sehr ankommt. Anfänglich macht dies einige Schwierigkeit, doch bald lernt man nach der Schnelligkeit mit der der Muskel sinkt oder steigt das nöthige Quantum der corrigirenden Lösung abzuschätzen. Hat man so das specifische Gewicht des Muskels erreicht, - wobei es, wie wir gleich sehen werden, auf absolute Genauigkeit zunächst noch nicht ankommt - so wird nun möglichst rasch der andere Gastrocnemius ans dem Körper entfernt und statt des ersten in die Flüssigkeit gebracht. Die specifischen Gewichte der beiden Gastrocnemien sind nie ganz genau einander gleich, schon deswegen weil es unmöglich ist von beiden genau gleichviel der specifisch schwereren Sehne abzuschneiden. Man kann daher den ersten Gastrocnemius nur zu einer annähernden Erreichung des richtigen specifischen Gewichts dèr Lösung für den zweiten Muskel verwenden und die endgiltige Abstimmung derselben erst dann vornehmen, wenn sich der zweite Muskel an Stelle des ersten in der Flïssigkeit befindet.

Die Reizelectroden bestanden aus einfachen Kupferdrähten mit einem isolirendem Kautschuküberzug, der an den Enden auf einer Strecke von einigen Millimetern entfernt worden war. Die beiden Drähte lagen der innern Wand des Messcylinders an, befanden sich diametral gegeniber und reichten so tief unter das Niveau der Flüssigkeit, dass die von Kautschuk entblössten Enden sich etwa in der Mitte des Cylinders befanden. Zwischen ihnen schwebte der Muskel völlig frei und ohne die Drähte zu berühren. Er nimmt dabei eine solche Lage ein, dass dasjenige Ende, welches in die Achillessehne iibergeht und daher schwerer ist, sich unten befindet. Ich will dieses Ende immer das Untere, das entgegengesetzte das Obere nennen.

Während ich nun den Muskel durch ein Fernrohr beobachtete 
varsetzte ich ihn in einen kräftigen dauernden Tetanus. Bei Beginn der Contraction bewegt sich das obere Ende des Muskels nach unten, das untere aber gleichzeitig nach oben. Während der Dauer des Tetanus bleibt der Muskel aber wieder in völliger Ruhe schwebend und ich habe in keinem Falle das geringste Sinken seines oberen Endes wahrnehmen können. Unterbrach ich die Reizung nach einigen Secunden, , so bewegte sich nun das obere Ende wieder nach oben und das untere nach unten. Dabei war jedesmal zu constatiren, dass das obere Ende sogar etwas höher hinaufkam als es vor der Reizung gewesen war. Der Muskel schwebte also nach dem Tetanus, wenn auch ohne die geringste Tendenz zum Steigen, immerhin doch etwas höher als vor demselben und man könnte auf die Annahme verfallen, der Muskel wäre während dieser Zeit etwas leichter geworden. Der Grund für dies geringe Steigen ist aber folgender: Wenn beim Beginn des Tetanus die Muskelfasern sich verkürzen, so sollte sich eigentlich, da der Muskel ja nirgends fixirt ist, das untere Ende um ebensoviel nach oben bewegen wie das obere Ende nach unten und es sollte diejenige Fläche - es braucht keine Ebene zu sein - welche dabei zwischen beiden Enden in Ruhe verbleibt in der Mitte des Muskels liegen. Nun ist aber das obere Ende des Muskels viel dicker als das untere und hat daher bei seiner Bewegung einen etwas grösseren Widerstand der Flüssigkeit zu überwinden als das letztere. In Folge davon rückt das untere Ende mehr nach oben als das obere nach unten d. b. die neutrale Fläche befindet sich in der oberen Hälfte des Muskels. Bei der nachfolgenden Erschlaffung des Muskels sollte sich dann freilich das obere Ende aus demselben Grunde weniger nach oben und das untere weiter nach unten bewegen und der Muskel solllte schliesslich seinen ursprïnglichen Stand wieder einnehmen, aber das Abfallen der tetanischen Curve erfolgt viel langsamer als das Ansteigen und bei der langsameren Bewegung ist der Widerstand, den die Flüssigkeit beiden Enden darbietet, weniger ungleich. Die neutrale Fläche liegt also bei der Erschlaffung tiefer als beim Entstehen des Tetanus and der Muskel muss daher nach jedem Tetanus etwas höher schweben. Un die Richtigkeit dieser Erklärung zu beweisen, habe ich in das obere Ende des Muskels ein kurzes Stückchen eines dünnen Platindrahtes versenkt, wonach sich der Muskel umkehrte und mit dem 
dickeren Ende nach unten schwebte. - Dabei musste natürlich das specifische Gewicht der Flüssigkeit entsprechend der Beschwerung des Muskels geändert werden. - Nun trat das Umgekehrte ein: nach jedem Tetanus stand der Muskel etwas tiefer als vorher.

Ich habe auch mit dieser Methode Säugethiermuskeln untersucht und auch am schwebenden Gastrocnemius des Meerschweinchens keine Spur von einem Sinken während des gleichmässigen Bestehens des Contractionszustandes sehen können. - Das specifische Gewicht des Meerschweinchen - Gastrocnemius betrug in einem Fall 1,0654. --

Empfindlichkeit der Methode.

Da man sich äber den Werth einer Methode nur durch die Bestimmung ihrer Empfindlichkeit ein Urtheil verschaffen kann, so habe ich grosse Sorgfalt auf die nachfolgende Untersuchung verwandt. Um zu erfahren, wie viel der Muskel an Volumen abnehmen muss, um ein deutliches Sinken innerhalb der möglichen Beobachtungszeit (Dauer des Tetanus) konstatiren zu können, braucht man nur das Gewicht zu bestimmen, welches man auf ihn legen muss, um ein genügend schnelles Sinken hervorzurufen. Diese Gewichte machte ich mir, indem ich von einem feinen Platindrabt kleine, etwa $1 \mathrm{~mm}$ lange Stiickchen mit der Scheere abschnitt. Die Dicke des Drahtes und die Länge der einzelnen Stiickchen wurden unter dem Mikroskop bestimmt. Das specifisehe Gewicht wurde aus der Schwere eines langen Stiuckes desselben Drahtes berechnet. Auf diese Weise ergab sich das Gewicht der einzelnen abgeschnittenen Drahtstückchen, von dem dann noch der Auftrieb in der Gummilösung, deren specifisches Gewicht also genau bestimmt werden musste, abzuziehen war. Es mag schwierig erscbeinen, diese winzigen Gewichte auf den Muskel zu bringen, es ist das aber nicht so schwer. Man muss nur eine glatte (nicht geriefte) Pinzette nehmen und dafür sorgen, dass die Innenflächen der Branchenenden ganz sauber sind. Man nimmt das Gewichtchen mit der Pinzette auf, bringt es unter die Gummilösung in eine kleine Entfernung iiber den Muskel und lässt es auf den Muskel durch Oeffnen der Pinzette fallen. War das Gewicht genügend gross, um ein Sinken des Muskels von der erforderlichen Schnelligkeit zu bewirken, so machte ich die Gegenprobe und nahm das Gewicht wieder fort. Dies ist dann in der That eine 
schwierigere Operation und wenn ich derartige Bestimmungen noch ein Mal zu machen bätte, so würde ich Eisendraht statt des Platindrahts anwenden und die Gewichte mit einem Magnet wieder abnehmen. Es gelingt zwar auch, die Gewichte mit der Pinzette zu fassen, aber man stösst dabei immer etwas gegen den Muskel, dieser kommt in schnelles Sinken und prallt schliesslich gegen den Boden des Gefässes an. Wenn er nun wieder steigt, hat man nicht die volle Garantie, dass dies seinen Grund in der Fortnahme des Gewichts hat. Ich machte daher immer noch eine Probe und liess dasselbe Gewicht noch ein Mal auf den Muskel fallen. Trat dann wieder ein Sinken wie früher ein, so wurde das Sinken und Steigen des Muskels der Belastung und folgenden Entlastung zugeschrieben und ich war sicher, dass mich die leicht eintretenden Fehlerquellen, die in Erwärmung, Diffusion, Abpralien vom Boden u. s. w. bestehen, nicht getäuscht hatten.

In einem Fall wog das kleinste Gewicht, das ein genügend schnelles Sinken des Muskels bewirkte, unter der Gummilösung 0,18 mgr. Das specifische Gewicht der Gummilösung betrug 1,0765. Hätte also der Muskel $0,168 \mathrm{cbmm}$ an Volumen bei der Contraction verloren, so wäre dies dentlich zu erkennen gewesen. In anderen Fällen waren die Zahlen sehr ähnliche, und die die Empfindlichkeit angebende Zahl der Cubikmillimeter schwankte nur zwischen 0,150 und 0,175 .

In Wasser sehwebender M uskel.

In der vorigen Methode, den Muskel in Gummilösung schweben zu lassen, ist die relative Unempfindlichkeit sehr auffallend. Sie rührt zum Theil daher, dass nicht nur ein Sinken des Muskels, sondern auch eine bestimmte Schnelligkeit dieser Bewegung gefordert wird. Wendet man nun zur Belastung kleinere Gewichte an als das oben angegebene Minimalgewicht, so werden die Zeiten, die der Muskel braucht, um ein geniigendes Stück - etwa einen balben Millimeter - zu sinken, zu gross, um eine gute Beobachtung zu gestatten, wenn das Sinken durch einen gleichmässigen Tetanus zu Stande kommen soll. Und zwar wachsen diese Zeiten in sehr schneller Progression, wenn man die Gewichte ganz allmählich verkleinert, so dass ich bei einem Gewicht von $0,10 \mathrm{mgr}$ iuberhaupt kein Sinken mehr wahrnehmen konnte. Offenbar hat auf diese Verhältnisse die Consistenz der Flüssigkeit den grössten 
Einfluss und um die Methode, den schwebenden Muskel zu beobachten, möglichst empfindlich zu machen, habe ich in einer neuen Anzahl von Versuchen den Muskel in Wassar schweben lassen.

Ich schmolz eine Quantität Wachs

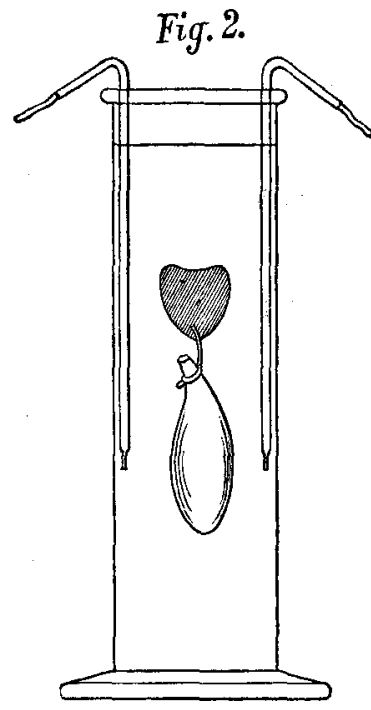

Figur 2. mit gleichen Theilen Talg zusammen und verriihrte in die Mischung eine geringe Menge Kienruss - die Schwarzfärbung hat den Zweck, die aufgelegten Gewichte leichter sichtbar zu machen. - Aus der erstarrten Masse formte ich einen birnenförmigen Körper, der an seinem dicken Ende schwach concav eingedrückt war, wie es die Figur 2 im Durchschnitt zeigt. Um die Achillessehne des Gastrocnemius legte ich eine Schlinge aus feinem Platindraht; sein eines Ende wurde kurz abgeschnitten, das andere versenkte ich in die Spitze des Wachskörpers. Auf diese Weise ist der letztere und der Muskel genügend fest miteinander verbunden und beide können zusammen in der Mitte des Wassers - wie immer mit einem Zusatz von $0,6 \%$ Kochsalz -- zum Schweben gebracht werden. Dazu misste der Wachskörper, dessen specifisches Gewicht etwas kleiner als das des Wassers ist, eine ganz bestimmte Grösse haben. Man erreicht aber das Schweben am leichtesten, indem man ihn etwas zu gross macht und ihn dann durch Auflegen kleiner Gewichte allmählich so lange beschwert, bis das System die gewünschte Stellung einnimmt. Die Gewichte bestehen in diesem Fall aus kleinen Stiickchen eines sehr dünnen aber massiven Glasfadens. Der schwebende Muskel wird in gleicher Weise wie im vorigen Falle tetanisirt und gleichzeitig durch ein Fernrohr beobachtet. Beim Beginn und beim Aufhören der Contraction wurden wieder die schon oben beschriebenen Bewegungen beobachtet. Aber auch bei Anwendung dieser Methode habe ich nie ein Sinken während des Tetanus gesehen. 
Empfindlichkeit der Methode.

Die Empfindlichkeit wurde in gleicher Weise wie bei Anwendung der Gummilösung bestimmt. Aus einer Glasröhre machte ich einen grösseren Glastropfen und bestimmte sein specifisches Gewicht. Aus derselben Glasröhre zog ich ferner einen massiven Glasfaden, von dem ich dann kleine Stücke mit einem Messer abdrückte, deren Dimensionen unter dem Mikroskop bestimmt wurden. Das kleinste Gewicht, welches ein genügend schnelles Sinken hervorbrachte, betrug $0,023 \mathrm{mgr}$. Das Wasser mit dem $0,6 \%$ Kochsalz hatte ein specifisches Gewicht von 1,004, aber dieser Unterschied von dem specifischen Gewicht des reinen Wassers ist so gering, dass er sich noch nicht auf die dritte Dezimalstelle geltend macht und die Gewichtsgrösse 0,023 drückt uns daher auch das Volumen aus, das der Muskel keinesfalls bei der Contraction verloren hat.

So ausgefuhrt wird also auch diese Methode recht empfindlich. Sie kann freilich in dieser Beziehung nicht mit dem Verfahren das Niveau in der Steigröhre zu beobachten wetteifern, sie wird aber immer den grossen Vorzug behalten, dass bei ihr nirgends Capillarkräfte auftreten wie bei allen iubrigen bis jetzt bekannten Methoden und dass sie daher sicher, soweit ihre Empfindlichkeit reicht ganz fehlerfrei ist.

Wiederholung des Valentin'schen Versuchs.

Bringt man nach Valentin's Angabe den Muskel zur Contraction während er sich unter Flüssigkeit befindet und mit einem Coconfaden an dem einen Wagebalken aufgehängt ist, so bewegt sich, wenn die Wage nicht arretirt ist, das untere Ende des Muskels viel weiter nach oben als das obere Ende nach unten, weil das letztere durch die Befestigung an der Wage an seiner freien Bewegung gehindert ist. Das obere Ende des Muskels muss bei seiner Bewegung nach unten die ganze Masse des Wagebalkens, der Schaalen und überhaupt aller Theile, die an der Bewegung der Wage theilnehmen, zu gleicher Zeit mit sich fortbewegen. Trotzdem wird regelmässig bei Beginn des Tetanus die Wage vom Muskel etwas herabgezogen und natürlich desto stärker je schwerer der Muskel ist und je schneller und grösser die Verkürzung ausfällt. Ein mïder Muskel bewirkt daher einen kleineren Ausschlag als ein frischer. 
Um nun zu sehen, ob während des Tetanus das specifische Gewicht des Muskels verändert ist, muss man die Rubelage der Wage bestimmen und zu dem Zweek mindestens 3 Umkehrungen der Zunge beobachten. Die langsamen Schwingungen einer empfindlichen Wage werden durch den in der Flüssigkeit mitschwingenden Muskel noch bedeutend verzögert. Der Muskel wirkt dämpfend, ohne jedoch die Bewegungen aperiodisch zu machen. Man braucht also zur Bestimmung der Rubelage viel Zeit und hierin liegt eine grosse Schwierigkeit, weil der Tetanus des Muskels nicht so lange gleichmässig andxuert. Man könnte versuchen die Zugwirkung des Mnskels bei der Verkürzung zu vermeiden und könnte den Aufhängepunkt des Muskels in die neutrale Fläche verlegen. Hierdurch würde bei einem länglichen Muskel wie dem Gastroenemius die Zugwirkung grösstentheils aufgehoben, ganz freilich erst dann, wenn der Aufhängepunkt zu gleicher Zeit der rubende Punkt der neutralen Fläche wäre. In diesem Fall würde aber die Aufhängung complicirt und zeitraubend werden. Näher liegt daher die Idee die Zugwirkung dadurch zu umgehen, dass man die Wage zunächst arretirt und erst während der Dauer des Tetanus frei lässt. Damit aber nur dann Scliwingungen der Wage auftreten, wenn sich wirklich das specifische Gewicht des Muskels geändert hat, miissen zugleich noch zwei Bedingungen erfültt sein. Erstens muss die Ruhelage der Wage für den Fall, dass der ruhende Muskel an ihr hängt, genau übereinstimmen mit derjenigen Lage der Wage, in der sie von der Arretirung frei gelassen wird. Zweitens darf durch das Wegschaffen der Arretirung keine Schwingung verursacht werden. Die erste Bedingung ist durch Verschiebung des Reiters zu erreichen und wie man leicht einsieht ist dadurch dann auch das Haupterforderniss zur. Erfïllung der zweiten Bedingung vorhanden. Man braucht dann nur geniugend langsam und gleichmässig die Arretirung zu beseitigen, so werden keine Schwingungen der Wage dadurch entstehen.

Mit Hilfe dieser letztbeschriebenen Methode erreichte ich es, die Schwierigkeiten, die sich bei der Benutzung der hydrostatischen Wage darbieten, zu umgehen. Der Muskel warde tetanisirt während die Wage arretirt war. Geich nach Beginn des Tetanus wurde sie vorsichtig frei gelassen und da ihre Ruhelage genau der Lage entsprach in der sie frei wurde, so konnte eine Schwingung der Wage überhaupt nur erfolgen, wenn das specifische Ge- 
wicht sich durch den Tetanus geändert hatte. Aber weder beim Gastrocnemius des Frosches, noch bei dem des Meerschweinchens trat die geringste Bewegung der Zunge ein.

Empfindlichkeit der Methode.

Die Wage hatte bei der in Frage kommenden Belastung eine Empfindlichkeit von 1,63 Theilstrichen $\left.{ }^{1}\right)$. Da ich mit Sicherheit eine Bewegung der Zunge um ein Zehntel eines Theilstriches erkannt haben würde, so haben die Muskel sicher weniger als $0,06 \mathrm{cbmm}$ beim Tetanus verjoren. Dabei lasse ich allerdings die Wirkung des Capillardrucks ganz unberïcksichtigt, aber es kommt mir in diesem Fall auch nicht auf eine ganz genane Bestimmung an. Valentin, der tibrigens die oben besprochenen Schwierigkeiten, die die Wage bei diesen Untersuchungen darbietet, nicht erwähnt, fand, dass der Muskel des winterschlafenden Murmelthiers um 1/1370 seines Volumens während des Tetanus abnimmt. Nehme ich das Volumen der von mir verwandten Muskeln zu durchschnittlich $1 \mathrm{cbcm}$ an, so hätten sie danach etwa $0,7 \mathrm{cbmm}$ verlieren müssen und meine Wage würde nach Wegnahme der Arretirung einen Ausschlag von etwa 2 Theilstrichen gemacht haben. Die neue Ruhelage wäre 1,14 Theilstriche von der bisherigen entfernt gewesen.

Wiederholung der Versuche von Erman, Marchand und Ed. Weber.

Wodurch das positive Resultat bei Erman's Versuch und bei dessen Wiederbolung durch Marchand und Ed. Weber zu Stande gekommen war, erschien völlig rätbselhaft. Keine von den Fehlerquellen, an die man denken könnte - wie eine mangelbafte Dichtung des Verschlusses, die Capillarität in der Steigröhre, in dem Gefäss zurückgebliebene Luft oder Gasausscheidung an den Electroden - keine derselben kann ein so plötzliches Sinken des Niveaus in der Steigröhre erklären. Sie veranlassen vielmehr ein Steigen des Niveaus oder verringern eine vorhandene Niveaubewegung. Für Marchand's und Ed. We ber's Versuch ist auch die von Joh. Müller herangezogene Möglichkeit, dass sich Luft im Innern des Aalschwanzes befunden haben könnte, durch das Sehlachten des Thieres unter Wasser völlig ausgeschlossen worden.

1) Vergl. Kohlrausch, Leitfaden der praktischen Physik, 1884, p. 28. 
So blieb hier nur die Hoffnung, bei einer Wiederholung des Versuchs vielleicht eine Fehlerquelle zu entdecken, die durch einfache Ueberlegung bei der Beurtheilung des Versuchs nicht zu finden war.

Zuerst überzeugte ich mich davon, dass auch bei der Contraction eines Aalschwanzes nicht die geringste Volumenabnahme wahrzunehmen ist. Die Versuchsanordnung war dabei im Wesentlichen dieselbe wie die von Erman und seinen Nachfolgern. Das Glasgefäss bestand aus einem $35 \mathrm{~cm}$ hohen Cylinder, dessen Durchmesser $54 \mathrm{~mm}$ betrug. Den Stopfen hatte ich mir aus bestem Kork eigens dazu schneiden lassen. In der Mitte wurde er von der Steigröhre durchsetzt, die am Ende zu einer Capillare mit einem Lumen von $0,26 \mathrm{~mm}$ ausgezogen war. In diesem letzten Punkt bin ich von Erman abgewichen und zwar aus demselben Grunde, den ich schon oben für meine eigenen Versuche geltend gemacht habe. Erman zog den einen Leitungsdraht durch die Steigröhre und liess sich dadurch den Vortheil, den die Capillare darbietet, entgehen. Meine beiden Leitungsdrähte gingen dagegen an zwei einander gegenüber befindlichen Stellen durch den Kork. Da es schwer ist dieselben durch den $5 \mathrm{~cm}$ hohen Kork wasserdicht $\mathrm{zu}$ ziehen, so kittete ich sie in enge Glasröhren ein uud steckte diese dann durch den Stopfen. Der eine Leitungsdraht war kurz und ragte nur wenig in den Cylinder hinein, der andere reichte dagegen bis auf den Boden. Vor dem Versuch wurde der Cylinder mit abgekochtem Wasser anfgefüllt und der Stopfen eine Zeit lang unter Wasser gelegt. Dabei wurde noch folgende Vorsichtsmaassregel angewandt. Ich entfernte mit einem Pinsel die kleinen Luftbläschen, die sonst leicht in den Poren und kleinen Vertiefungen des Korks sitzen bleiben und dadurch diese Stellen vor der Benetzung schïtzen. Sind aber einmal die Wände dieser Vertiefungen nass geworden, so kann man nachher den Pfropfen auf den ganz mit Flüssigkeit gefülten Cylinder aufsetzen ohne dass von neuem an diesen Stellen Luftblasen zurückbleiben.

Ich verwandte zu dem Versuch zwei Aale, von denen der erste nur zur Vorbereitung des eigentlichen Versuchs diente. Das zweite Thier wurde unter abgekochtem Wasser geschlachtet und der hintere Körperabschnitt bis zum Beginn der Leibeshöhle durch einen einfachen Schnitt vom übrigen Körper getrennt. Nur der Schwanz wurde zum Versuch verwandt. Das freie Ende der Wirbelsäule befreite ich auf anderthalb Centimeter von der umgeben- 
den Muskulatur, um den kürzeren der im Kork befindlichen Leitungsdrähte daran befestigen zu können. Er wurde eine Strecke weit ins Rückenmark hineingesteckt, um das frei präparirte Ende der Wirbelsäule herumgebogen und schliesslich in dieser Lage mit einer Flachzange fest angedrückt. Das Ende des längeren Leitungsdrahts zog ich durch ein Loch, das ich mit einem Pfriemen durch das äusserste Schwanzstïck gebohrt hatte und drehte es dann zu einer Oese zusammen. Nun wurde der Aalschwanz in den Cylinder gebracht und dieser möglichst fest mit dem Pfropfen verschlossen. Doch war für die Befestignng des letzteren noch in besonderer Weise gesorgt. Es war ein starker Messingdrabt oben um den Cylinder unmittelbar unter dern Rande herumgelegt und von diesem gingen zwei Drahtbügel aus, die sich rechtwinklig über dem Pfropfen kreuzten. Die Bügel bildeten an einer Stelle Oesen und durch weiteres Umdrehen derselben und dadurch bedingtes Aufwickeln des Dralites um sich selbst wurden die Bügel gegen den Pfropfen und dieser in den Cylinder gepresst. Das verdrängte Wasser floss dabei aus der Steigröhre heraus.

War alles so weit vorbereitet, so musste nur noch das Niveau in der Steigröhre in einen mittleren Abschnitt der Capillare gebracht werden, was durch Einführen und Herausziehen eines diinnen Drahtes geschah. Man verdrängt auf diese Weise aus der Capillare soviel Wasser als nöthig ist. Dann wurde der Aalschwanz durch Inductionsströme (Schlitteninductorium mit 50-60 inducirten Strömen in 1 Sec.) gereizt. Er bewegt sich dabei mit grosser Heftigkeit, indem wellenförmige Bewegungen in der Richtung zum Schwanzende ablaufen. Das Niveau in der Steigröhre blieb aber dabei unverrückt stehen, auch als ich es durch ein Mikroskop beobachtete.

Wenn aber der Aalschwanz sein Volumen bei der Contraction nicht ändert, so bleibt nun zu erklären, wodurch in den Versuchen Ermans und seiner Nachfolger das Sinken des Niveaus in der Steigröhre veranlasst wurde. Der Grund dafür besteht in einer ungenügenden Befestigung des Pfropfens. Ich wandte, wie geschildert, zwei Drahtbugel an, um den Pfropfen in den Cylinder zu pressen, weil ich schon im Voraus wusste, dass die Befestigung des Pfropfens schwierig sein witrde. Dies ist aber von meinen Vorgängern unterlassen worden und darauf ist offenbar die Verschiedenheit unserer Resultate zurückzuftuhren. Als ich nach An- 
stellung des obigen Versuchs die Drabtbügel wieder entfernte und nun von Neuem reizte, da bekam auch ich ein starkes Sinken des Niveaus, welches sogar mehrmals über $10 \mathrm{~mm}$ betrug. Es erklärt sich das nun folgendermaassen.

Ein Pfropfen mit einem so grossen Durchmesser $(5,5 \mathrm{~cm})$ ist schon allein wegen seiner Grösse sehr schwer fest in das Gefäss einzudrücken. Es kommen aber noch zwei Umstände hinzu, die seine Befestigung erschweren. Erstens schneidet man so grosse Pfropfen konisch zu, wodurch die fest an das Glas angreifende Zone verhältnissmässig klein wird und sich seine Neigung zuräckzugleiten vermehrt. Zweitens ist das Eindrücken des Pfropfens in diesem Fall sehr erschwert, weil die Steigröhre verhindert die Hand über ihn zu legen und ihn in der bekannten Weise unter Druck einzudrehen während man ibn zwischen Fingerspitzen und Daumenballen einklemmt. Wenn aber der Pfropfen nicht geniigend in den Cylinder gepresst wird, so bedarf es nur einer kleinen Erschitterung, um die Reibung zu überwinden, die ihn am Zuriickgleiten hindert. Reizt man unter solchen Umständen den Aalschwanz, so genügt die Erschütterung, die er bei seiner Contraction dem Pfropfen ertheilt, um denselben etwas zu lockern und die Folge davon ist natürlich, dass das Niveau in der Steigröhre sinkt. Ich konnte dasselbe beobachten, wenn ich den Pfropfen durch Aufschlagen mit einem ganz kleinen Hammer oder einem Finger erschütterte.

Der Stoss den der Aalschwanz auf den Pfropfen ausübt, hat ubrigens eine besonders grosse Wirkung, weil das Aalstiick - das Kopfende oben vorausgesetzt -- in derselben Richtung gegen den Stopfen stösst, in der dieser aus dem Gefäss zu gleiten strebt. Beim Aufschlagen mit dem Hammer kommt dagegen diese Bewegung direct nicht zur Wirkung, sondern nur die entgegengesetzte, die durch die Elasticität des Korks hervorgebracht wird.

Wenn nun diese Erklärung für das Sinken des Niveaus bei der Contraction des Schwanzes die richtige war, so musste man auch ein Steigen des Niveaus in analoger Weise hervorbringen können. Dazu musste dann eigentlich der Pfropfen von innen her gegen die Oeffunng des Gefässes gepresst werden und man hätte daza ein besonders gestaltetes Gefäss verwenden mïssen.

Ich erreichte jedoch dasselbe in einfacherer Weise. Die Drahtbügel wurden wieder über den Pfropfen gelegt und nachdem 
ich sie ziemlich fest angezogen hatte, versuchte ich den Pfropfen mit aller Gewalt aus dem Cylinder herauszudriucken. Das ist dann nur soweit möglich, als sich der Pfropfen zwischen Bügel und driickendem Finger einzwängen lässt. Bei dieser Procedur sinkt natürlich das Niveau in der Steigröhre. Lässt man nun aber mit Drücken ganz allmählich nach, so steigt nun das Niveau, denn der Pfropfen schiebt sich vermöge seiner Elasticität wieder tiefer in das Gefäss hinein, bis ihn die Reibung am weiteren Eindringen hindert. In diesem Zustand des Stopfens kann man in genau derselben Weise wie in der oben angegebenen, also auch durch Reizung des Aalschwanzes, das Niveau zum Steigen bringen. Der Pfropfen hat jetzt die Tendenz tiefer in das Gefäss zu gieiten und jede Erschütterung von einiger Stärke ermöglicht diese Bewegung.

In diesem eigenthümlichen Verhalten des Pfropfens glaube ich also eine Erklärung für die abweichenden Resultate von Erman, Marchand und Ed. Weber gefunden zu haben und jeder, der den Versuch wiederholt, wird sich leicht davon überzeugen, wie schwer es ist obne Bügel diese Fehlerquelle zu vermeiden. Das Steigen des Niveaus, das Ed. Weber in den Pausen zwischen der Reizung sah, kann leicht auf allmähliche Erwärmung der Flüssigkeit bezogen werden, und wenn die Abfälle des Niveaus bei jeder folgenden Reizung kleiner wurden, so hatte das offenbar darin seinen Grund, dass der Pfropfen dureh jede Erschtitterung seiner Gleichgewichtslage näher kommt.

Die von Harless angewandten Methoden.

Das Interesse, welches sich an die Versuche von Harles s knuipft, bezieht sich nur auf die dabei benutzten Methoden, nicht auf die Resultate selbst. Die Fehlerquellen waren offenbar sehr gross, es dürfte aber unmöglich sein ihre Grösse anch nur ungefäbr in Zahlen anzugeben. Ich habe deshalb die Versuche weder wiederholt, noch sie in der unten angefitgten Zusammenstellung der Methoden und ihrer Fehlerquellen mit aufgefuhrt. Nur den einen gleich zu besprechenden Versuch habe ich in ähnlicher Weise wie Harless angestellt, um über seine Brauchbarkeit ein sicheres Urtheil zu gewinnen. Es ist dies der Versuch, bei dem zwei communicirende Röhren zur Verwendung kommen; in der einen befindet sich Wasser, in der andern Steinöl. Die beiden Flüssigkeiten stossen in dem engen Verbindungsstiick der Röhren 
zusammen und aus der Verschiebung dieser Grenze wird auf eine Niveauveränderung in den Röhren geschlossen. In dem Wasser der einen Röhre kann der zu untersuchende Muskel zur Contraction gebracht werden; seine Volumenschwankungen bewirken ein Steigen oder Sinken des Niveaus in derselben Röhre und dadurch auch eine Verschiebung der Grenze zwischen dem Wasser und dem Steinöl in der Communicationsröhre. Herman $n^{1}$ ) sagt von dieser Methode, sie scheine „weniger exact als die Erman-Weber'sche; die Volumänderung wirkt nicht einmal in voller Grösse verschiebend." Dieser letztgenannte Umstand ist freilich nicht günstig für die Empfindlichkeit der Methode, aber die eigentliche Fehlerquelle liegt in der ungleichen Benetzung der Glaswand durch das Wasser. Man kann sich davon in folgender Weise sehr leicht iiberzeugen. Man bringe in das Wasser eine feine Capillare, die durch einen Gummischlauch mit einem kleinen Druckgefäss in Verbindung steht. Richtet man es nun so ein, dass durch die Capillare nur ganz langsam aber völlig continuirlich Wasser aus dem Druckgefäss in die communicirende Röhre fliesst, so sollte sich, wenn die Methode brauchbar wäre, die Grenze zwischen Wasser und Steinöl langsam aber ebenfalls continuirlich verschieben. Dies ist aber keineswegs der Fall. Ebenso ruckweise wie das Niveau an der Glaswand in Folge der wechselnden besseren oder schlechteren Benetzung steigt, verschiebt sich auch die betreffende Grenze.

Bei der zweiten von $\mathrm{Harless}$ angewandten Methode soll der Muskel von einer starren Masse uberall umgeben werden, die verhindert, dass sich irgend ein Querschnitt des Muskels bei der Contraction vergrössern kann. Es ist klar, dass unter solchen Umständen eine noch zu Stande kommende Verkürzung eine Volumabnahme des Muskels beweisen würde. Hermann${ }^{2}$ ) bezeichnet diese Methode sehr richtig als ebenso umständlich wie wenig beweisend und E. $d u$ B o is-Reymon $d^{3}$ ) bat auf die Fehlerquelle aufmerksam gemacht, die das Bestreichen des Muskels mit dem Oel behufs besseren Anschmiegens der Form mit sich bringt.

1) a. a. 0 .

2) a. a. 0 .

3) Arch. f. Anat. u. Physiol. 1873, p. 605 od. Ges. Abh. II, p. 472. 
Ich will einige von den Bedingungen aufä̈hlen, von deren Erfüllung die Brauchbarkeit dieser Methode abhängt, die aber von Harless nicht beachtet worden sind. Man wird jedoch nicht daran denken dürfen danach die Methode verbessern zu wollen. Denn wahrscheinlich lassen sich diese nöthigen Bedingungen iuberhaupt nicht genügend erfüllen und selbst wenn dies möglich wäre, so würde die Methode wegen ihrer grossen Umständlichkeit und dabei sehr geringen Empfindlichkeit ganz zu verwerfen sein. $\mathrm{Zu}$ den Bedingungen gehören: Die den Muskel umgebende Masse muss seinen Kräften gegenüber vollständig starr und unnachgiebig werden. Sie muss flüssig die Temperatur des Muskels haben und auch nach dem Erstarren immer behalten. Beim Erstarren selbst darf sich ibr Volumen weder vergrössern noch verkleinern. Auch ist darauf zu achten, dass dem Muskel kein Wasser durch die Masse entzogen werde. Die von Harless angewandte Cacaobutter scheint nur der letzten von den angefiihrten Forderungen entsprochen zu haben.

\section{Zusammenstellung der Fehlerquellen und der Em-} pfindlichkeit der verschiedenen Methoden.

Als Empfindlichkeit der Methoden werde ich in nachfolgender Zusammenstellung dasjenige Volumen (in abgerundeter Zahl) an. geben, das als Zuwachs oder Abnahme des Muskelvolumens einen genügenden Ausschlag gibt. Wo diesbezügliche Angaben der Autoren fehlen, habe ich die Empfindlichkeit zu schätzen versucht, wobei ich möglichst zu Gunsten der früheren Methoden gerechnet habe. Ich stelle die Zahlen zusammen, weil der Vergleich der Empfindlichkeiten interessant ist, wenn auch ein Theil der Zahlen nur auf Sehätzung beruht.

1. Die Erman-Marchand-Weber'sche Methode. Eine Fehlerquelle ist sehr leicht durch das Gleiten des Pfropfens gegeben, sie wird vermieden indem man über den Pfropfen Bügel legt oder eingeriebene Glasstöpsel verwendet. Die Empfindlichkeit dürfte $0,5 \mathrm{cbmm}$ nicht überstiegen baben.

2. Kühne's Aräometer-Methode. Die Fehlerquelle besteht in dem Capillardruck und in Unregelmässigkeit bei der Benetzung. an der Stelle, wo der Aräometerhals durch das Flüssigkeitsniveau geht. Die Empfindlichkeit des benutzten Aräometers ist vom Autor nicht angegeben worden. Nehme ich aber an, dass ein Ein- 
sinken des Aräometers um $0,5 \mathrm{~mm}$ schon zuverlässig auf eine Aenderung des Muskelvolumens schliessen liess und veranschlage ich den Durchmesser der Aräometerkugel auf $5 \mathrm{~cm}$, so würde sich eine Empfindlichkeit von $0,3 \mathrm{cbmm}$ ergeben.

3. Valentin's Verwendung der Wage. Die Fehlerquelle liegt in der Uebertragung der Muskelbewegungen auf die Wage; wie sie zu vermeiden ist wurde oben ausfïhrlich besprochen. Die Empfindlichkeit betrug etwa $0,1 \mathrm{cbmm}$.

4. Meine Methoden: a) die verbesserte Erman'sche Methode. Die Fehlerquelle, die in dem Capillardruck zu suchen wäre, ist verschwindend klein, falls die Capillare sorgfältig ausgezogen ist. Die Empfindlichkeit betrug 0,0001 cbmm.

b) Der in Gummilösung schwebende Muskel. Keine Fehlerquelle, dabei eine Empfindlichkeit von $0,2 \mathrm{cbmm}$.

c) Der in Wasser scbwebende Muskel. Keine Feblerquelle und eine Empfindlichkeit von 0,03 cbmm.

\section{A n h a n g.}

Die Fehlerquelle der eingedrungenen Luft.

Johannes Müller hat bekanntlich darauf aufmerksam gemacht, dass durch Eindringen von Luft in die Blutgefässe des Muskels eine Volumenabnahme bei der Contraction vorgetäuscht werden könnte. Er stellte sich dabei offenbar vor, dass die eventuell eingetretene Luft bei der Contraction des Muskels comprimirt würde. Ich habe auf diese Fehlerquelle mein besonderes Augenmerk gerichtet.

Damit es zu einer Compression von Luft kommen kann, müssen nothwendiger Weise zwei Bedingungen erfült sein. Erstens muss natiurlich Luft in die Gefüsse eingedrungen sein, zweitens darf aber auch die Luft bei der Contraction des Muskels nicht wieder heraus können. Was die erste Bedingung betrifft, so ist ein solches Eindringen von Luft in die Arterien grösserer Thiere, bei denen sie eine gewisse Wandstarre haben, gewiss leicht mög- 
Ewald: Aendert sich das Volumen eines Muskels bei der Contraction. 239

lich. Dagegen wird die Luft schon schwer in die Venen auch grösserer Thiere dringen, falls man das Präparat nicht unter besonders ungünstige Bedingungen bringt. Bei kleineren Thieren wie z. B. bei Meerschweinchen, Aalen und Fröschen collabiren aber Arterien und Venen derart, dass es ganz besondere Schwierigkeiten macht kleine Luftmengen in die Gefässe einzuführen. Man muss dann schon eine Spritze, eine Pipette oder dergleichen anwenden. Gesetzt aber auch, es sei eine Quantität Luft in irgend eine Arterie gedrungen, so wird dieselbe doch immer bei der Contraction des: Muskels denselben Weg rückwärts aus der Arterie wieder herauswandern können, den sie vorwärts hineingekommen ist. Dass der Muskel bei seiner Contraction eher die Arterienstämme versehliessen sollte als die Verästelungen, ist wohl gar nicht denkbar. Ich habe diese Verhältnisse auch experimentell geprifft. Mit Hülfe einer feinen Pipette brachte ich $1-2$ cbmm Luft in die Arterie des Gastroenemius vom Frosch und vom Meerschweinchen. Die Luft blieb grösstentheils in der Arterie, als ich den Muskel tetanisirte und meist wurde nur ein ganz kleines Bläschen ausgetrieben ${ }^{1}$ ). Das Volumen des Muskels änderte sich aber auch unter diesen Umständen nicht im Geringsten.

So bliebe also für eine mögliche Compression nur noch Luft übrig, die in Venen eingedrungen ist und durch die Venenklappen verhindert wird, nach Aussen zu entweichen. In diese dünnwandigen Gebilde kleiner Thiere dringt aber unter gewöhnlichen Verbältnissen keine Luft oder doch nur so wenig, dass es mir nicht möglich erscheint, die angebliche Volumenverminderung des Muskels bei der Contraction durch Compression von eingedrungener Luft zu erklären.

1) Die Frage, ob der Hohlraum der Arterien bei der Contraction des Muskels verkleinert wird oder nicht, hängt mit der Frage, ob die Muskelsubstanz ihr Volumen dabei ändert, gar nicht direct zusammen. Nach der obigen Erfahrung scheint sich der Hohlraum der Arterien etwas zu verkleinern. 